\title{
Category-level contributions to the alphanumeric category effect in visual search
}

\author{
J. PAUL HAMILTON, MICHELLE MIRKIN, and THAD A. POLK \\ University of Michigan, Ann Arbor, Michigan
}

\begin{abstract}
Do letter and digit recognition depend on the same or different cognitive mechanisms? Letters are detected more quickly among digits than among letters; likewise, digit search is facilitated when distractors are letters, as opposed to digits. This effect suggests that different mechanisms underlie recognition of these two categories. There are, however, systematic physical differences between letters and digits that might account for the effect. We used target and distractor stimuli that facilitated within-category search when inverted, and category identity was, thereby, attenuated. However, in conditions of upright search, in which category identity was more salient, between-category search was more efficient for the same stimuli. These findings suggest that letter and digit recognition are, at least to a degree, functionally independent.
\end{abstract}

The category effect in visual search is noted when search for a target from one category is more efficient when the target occurs among distractors from another category than when it occurs among distractors from its own category. Such an effect has been most frequently noted and studied within the domain of orthography: Letter (and digit) targets are found more efficiently among digit (and letter) distractors than among other letters (digits) (Brand, 1971; Egeth, Atkinson, Gilmore, \& Marcus, 1973; Egeth, Jonides, \& Wall, 1972; Jonides \& Gleitman, 1972; Neisser, 1967; Sperling, Budiansky, Spivak, \& Johnson, 1971). This so-called alphanumeric category effect is of potential interest to cognitive psychologists for two reasons. First, it is indicative of a dissociation in the cognitive architecture between perception of digits and perception of letters and suggests that they may rely on partially independent mechanisms. Second, it points to the possibility that learned distinctions between stimulus classes can have effects at preattentive levels of vision (Neisser, 1967; Treisman \& Gormican, 1988). Both of these points, though, are controversial.

Some researchers have argued that the effect is categorical; that is, it arises because the categories of letters and digits are recognized differently. Others have argued that differences in the visual features that make up letters and digits can account for the effect (e.g., digits have more curves than do uppercase letters; Keren, 1977).

Jonides and Gleitman (1972) have provided some of the most compelling evidence that the effect is categorical. They found that presenting the ambiguous target stimulus $\mathrm{O}$ as a letter ("oh") facilitated search among digit distrac-

M.M. is now at the Department of Psychology, Pepperdine University. We thank Patricia Reuter-Lorenz and John Jonides for their insightful contributions to the research presented here. Correspondence concerning this article should be addressed to J. P. Hamilton, Department of Psychology, Stanford University, 450 Serra Mall, Jordan Hall: Building 420, Stanford, CA 94305 (e-mail: paulhami@stanford.edu). tors, in comparison with letter distractors, whereas presenting it as a digit ("zero") had the opposite effect. Thus, search efficiency was significantly influenced by manipulating the category of the target even when its visual features were held constant, a finding strongly in favor of a category-level hypothesis. Ingling (1971) achieved similar results, but by matching the letters and digits for physical properties. Duncan (1983b) used a partial report task in which participants saw arrays consisting of all letters, all digits, a letter among digits, or a digit among letters and were then asked whether a probe item was present within the array. The letters and the digits were closely matched in terms of such features as curves, closure, and vertical lines. The participants were more accurate for digit probes when the array consisted of a digit among letters than when it consisted of just digits; likewise, the participants were more accurate for letter probe trials when the array was a letter among digits than when it was all letters. Duncan (1983b) also showed that the magnitude of the effect was the same when the letters and digits were artificially manipulated so that reliable physical differences were present between them. Thus, manipulating stimulus categories had an effect, but manipulating visual features did not. Finally, Dixon and Shedden (1987) obtained behavioral evidence for the similarity of stimuli used. They obtained reaction time and accuracy data for pairwise, same-different judgments of stimuli within and across categories that indicated that physical similarity was equated for these stimuli. Using the same stimuli, they obtained the standard alphanumeric category effect.

In contrast to these studies, there are a number of other studies whose results argue against the categorical hypothesis. Krueger (1984) used letter and digit stimuli that were matched for apparent similarity ( $P$ matched with 9 , 2 matched with $Z$, etc.) and found no category effect. In another experiment, he used the same stimuli and mirrorreversed them, thereby minimizing their familiarity and category salience, while maintaining similar physical attri- 
butes. With these unfamiliar stimuli, participants did show a category effect. The author contended that showing a category effect only when category status was not salient was convincing evidence against a category-level hypothesis.

Corcoran and Jackson (1979) had participants search for a C, 6,4 , or A among curved or angular distractors. Response time was affected by degree of physical resemblance between targets and distractors ( $C$ was found more quickly among angular than among curved distractors), and not by whether or not they were from the same category. Similarly, Cardosi (1986) reported that the magnitude of the alphanumeric effect changed significantly with target identity and was negatively correlated with the degree of similarity between targets and distractors. Both of these effects, she argued, support a physical differences hypothesis. Finally, Duncan (1983a) attempted twice to replicate the findings of Jonides and Gleitman (1972), using the ambiguous stimulus "O," and achieved null results both times.

To date, all of the studies in favor of the category-level hypothesis have attempted to show that the effect remains when physical differences between letters and digits are controlled. In the Ingling (1971) and Duncan (1983b) studies, control was incomplete. Matching 2 with $Z$ and 6 with $G$, for example, still results in letters' possessing more linear features than do digits. Over many trials, even slight statistical differences in feature properties between letters and digits could be detected in early vision and could sum to an apparent category-level effect. The study reported by Dixon and Shedden (1987), in which evidence of stimulus similarity was obtained, did so in the context of pairwise stimulus comparisons that might not extend to conditions of visual search. The "oh-zero" phenomenon of Jonides and Gleitman (1972) stands as the only example of perfect control of physical differences between stimulus categories, and it has not been replicated (Duncan, 1983a).

Studies that support the physical differences hypothesis have shortcomings as well. For example, the Cardosi (1986) study showed only that the magnitude of the alphanumeric effect was variable depending on the physical properties of the stimuli used. It did not show that category-level effects are not present as well. It is possible that both category-level effects and effects due to physical differences can operate simultaneously and that the category-level contribution can be attenuated or eliminated depending on the physical characteristics of the stimuli used. The same criticism can be levied against the Krueger (1984), Corcoran and Jackson (1979) and Duncan (1983a) studies. Their null effects might be due to remaining physical differences between letters and digits that favor within-category search and overcome a weaker, but real, between-category effect.

In this experiment, we tested whether a category-level effect would remain once physical differences between letters and digits had been controlled empirically within the context of visual search. If they did, we would have obtained evidence in favor of a category-level contribu- tion to the alphanumeric category effect; if not, a physical differences hypothesis would be supported.

\section{METHOD}

\section{Participants}

Thirty-eight University of Michigan undergraduates participated in this study in partial fulfillment of the research participation requirement for an introductory psychology course. Nine of these participants were eliminated from the analysis because their error rates exceeded a threshold of $3 \%$ (although including their data in the analysis had no significant effect on the results).

\section{Stimuli}

The participants searched for letter targets within a clocklike array -4 in. in diameter - of six elements that contained either other letters or digits. The letters and digits were presented in 24-point Helvetica font. The first objective of the study was to manipulate the salience of the physical properties of both the letters and digits. To make the physical properties of both the targets and the distractors more salient, we inverted them (see Figure 1). The technique of presenting stimuli in an unusual orientation, in order to attenuate category or identity information, has been used successfully by other researchers (e.g., Krueger, 1984).

Our next objective was to account for any physical differences between the letters and the digits that could give rise to the alphanumeric effect. We did so by using letter targets that we judged to be physically similar to the digit distractors and physically dissimilar to the letter distractors. We used letter targets that were relatively curved $(\mathrm{C}$ and $\mathrm{U})$ and put them among rectilinear letter distractors $(E, F, T, H$, and $L$ ) and curved digit distractors $(2,3,5,6$, and 7 ), which, as a group, have more curves. Note that for inverted search trials, all the characters were flipped about their horizontal axes, with the exception of $\mathrm{C}, \mathrm{E}$, and 3 , which were flipped about their vertical axes, and $\mathrm{H}$, which was rotated $90^{\circ}$ (see, e.g., Figure 2).

\section{Procedure}

Each participant did blocks of 128 trials of each of the four types of search detailed above: inverted between category (IBC), inverted within category (IWC), upright between category (UBC), and upright within category (UWC). Blocks were counterbalanced across participants to control for order and practice effects. Trials for each search type were blocked, as opposed to being intermixed, since the alphanumeric effect has occurred more reliably when trials have been blocked (Gleitman \& Jonides, 1978). One third of all the trials were target-absent catch trials. The target ( $\mathrm{C}$ or $\mathrm{U}$ ) was chosen randomly for each trial, and each target appeared the same number of times. All the distractor stimuli from a particular category appeared

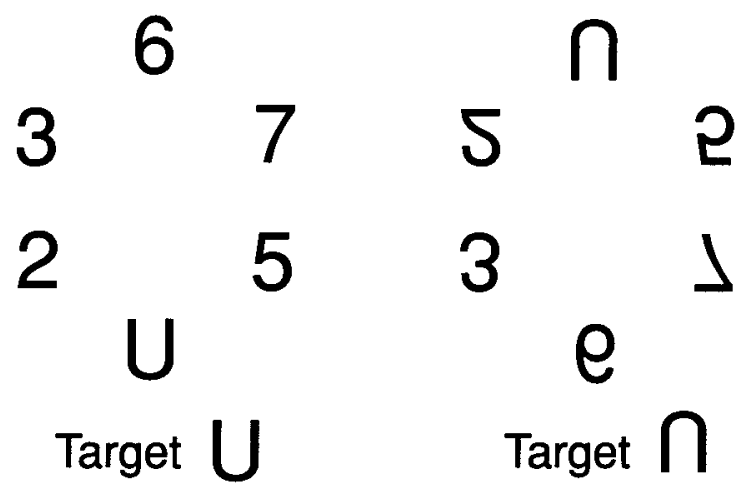

Figure 1. Sample of upright and inverted search trials. 


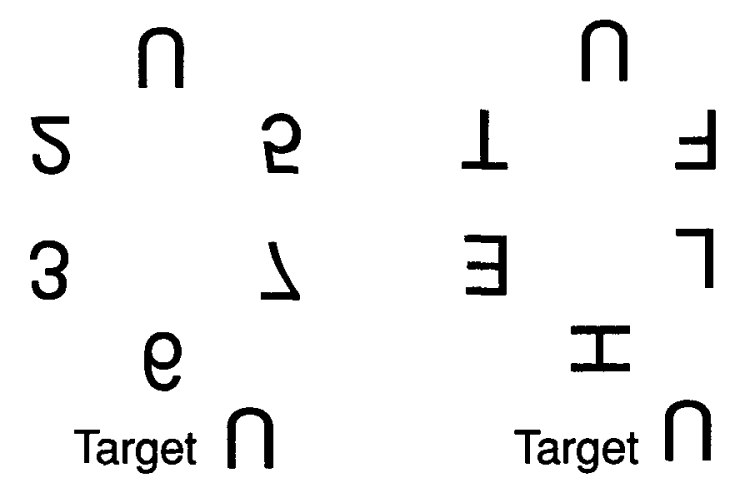

Figure 2. Sample of stimuli used to facilitate within- versus between-category search for inverted search trials.

on each of the trials. For the catch trials, one of the distractor stimuli was chosen at random to be repeated within the search array.

The trials were structured as follows. First, the target stimulus appeared in the center of the screen for encoding. The participants were instructed to press the space bar once they were ready to advance to the search portion of the trial. For the search portion of the trial, the participants were instructed to find the target among the distractors as quickly as possible while maintaining a high degree of accuracy and to press the right-middle finger key (" $k$ " key) on target-present trials and the left-middle finger key ("d" key) on target-absent trials.

\section{RESULTS}

Figure 3 shows the results. Two planned contrasts were conducted in accordance with the aims of the study. We assessed the effect of the search type factor at each level of the stimulus orientation factor. First, we compared between- with within-category search for inverted stimuli, which emphasized physical, over category, attributes during search. For these stimuli, the direction of the normal alphanumeric effect reversed. The mean reaction time and standard error ( $S E)$ for IWC search was $546 \mathrm{msec}(S E=$ $17.7 \mathrm{msec})$, as opposed to $560 \mathrm{msec}(S E=16.6 \mathrm{msec})$ for IBC search $[F(1,27)=4.33, p<.05]$.

Next, we assessed the effect of search type for upright stimuli. When the stimuli were presented upright, making category membership more salient, the usual category effect was reinstated, despite the fact that withincategory search was favored by the physical attributes of the stimuli. The UBC search mean was $525 \mathrm{msec}(S E=$ $15.3 \mathrm{msec}$ ) versus $551 \mathrm{msec}(S E=18.4 \mathrm{msec})$ for UWC search $[F(1,27)=13.83, p<.05]$.

\section{DISCUSSION}

These data support the hypothesis that the advantage of between-category over within-category search occurs, at least in part, due to the categorical distinction between letters and digits, and not just because of their physical particularities. Using stimuli whose physical attributes actually favor within-category search, as indicated by the within-category search advantage for inverted stimuli, a category effect was nonetheless obtained when the same stimuli were upright.

These data provide a more convincing demonstration of a category-level effect than have the results of previous studies for two reasons. First, our attempt to control for physical differences between letter and digit sets was empirically validated, as opposed to being based on an estimation of control for physical attributes. Second, we obtained evidence of control of the physical features of letter and digit stimuli in the context of visual search.

These data support the notion of category-level contributions to the alphanumeric category effect. The presence of this category-level influence suggests that the dissociation noted between letter recognition and digit recognition reflects a distinction between them that goes beyond the physical dissimilarities of letters and digits and, instead, reflects a dissociation in the mechanisms underlying the processing of the two stimulus categories.

Beyond their implications regarding the dissociability of letter and digit processing, the results presented here indicate that learned distinctions between artificial clasises of stimuli have significant effects on visual attention that are likely to act at preattentive levels of processing. Results from Esterman, Prinzmetal, and Robertson (2004) support the formulation that alphanumeric categorization happens early in visual processing and can affect subsequent processes at the threshold of attention-specifically, the conjoining of object identity and color. They found that illusory conjunctions in which the target stimulus was incorrectly seen as being the same color as $\mathrm{O}$ were more likely when the ambiguous character $O$ was contextually manipulated to be of the same alphanumeric category as a

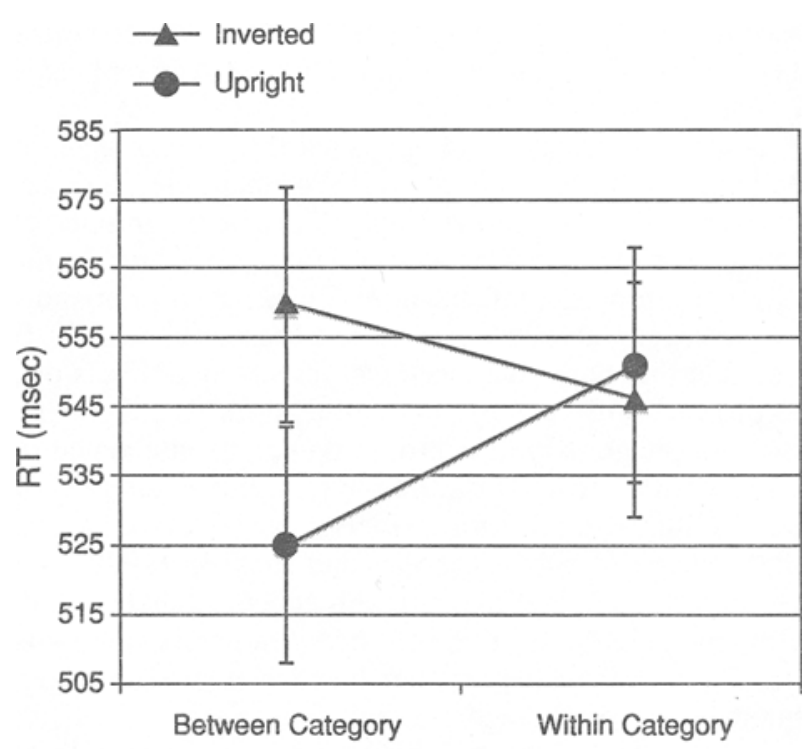

Search Type

Figure 3. Mean reaction times (RTs), with standard error bars, for correct responses on target-present trials for the four permutations of the search type and stimulus orientation factors. 
target stimulus than when $\mathrm{O}$ and the target stimulus were of different categories (Esterman et al., 2004).

The work presented here begs further investigation into how learning impacts preattentive visual processing, as well as into the pervasiveness of these effects in the visualprocessing hierarchy. Renewed interest in the effects of learning on visual processing (e.g., Haijiang, Saunders, Stone, \& Backus, 2006; Witthoft \& Winawer, 2006) and further refinements in methodology are likely to yield informative results.

\section{REFERENCES}

BRAND, J. (1971). Classification without identification in visual search. Quarterly Journal of Experimental Psychology, 23, 178-186.

CARDOSI, K. M. (1986). Some determining factors of the alphanumeric category effect. Perception \& Psychophysics, 40, 317-330.

Corcoran, D. W. J., \& JACKsON, A. (1979). Flexibility in the choice of distinctive features in visual search with blocked and random designs. Perception, 8, 629-633.

DiXON, P., \& SHEDDEN, J. M. (1987). Conceptual and physical differences in the category effect. Perception \& Psychophysics, 42, 457-464.

DUNCAN, J. (1983a). Category effects in visual search: A failure to replicate the "oh-zero" phenomenon. Perception \& Psychophysics, 34, 221-232.

DUNCAN, J. (1983b). Perceptual selection based on alphanumeric class: Evidence from partial reports. Penception \& Psychophysics, 33, 533-547.

Egeth, H., Atkinson, J., Gilmore, G., \& Marcus, N. (1973). Factors affecting processing mode in visual search. Perception \& Psychophysics, 13, 394-402.
EgEth, H., JoNides, J., \& WALL, S. (1972). Parallel processing of multielement displays. Cognitive Psychology, 3, 674-698.

Esterman, M., Prinzmetal, W., \& Robertson L. (2004). Categorization influences illusory conjunctions. Psychonomic Bulletin \& Review, $11,681-686$.

HaiJiang, Q., Saunders, J. A., STone, R. W., \& Backus, B. T. (2006). Demonstration of cue recruitment: Change in visual appearance by means of Pavlovian conditioning. Proceedings of the National Academy of Sciences, 103, 483-488.

INGLING, N. (1971). Categorization in visual information processing. Dissertation Abstracts International, 32, 6B.

JoNIDES, J., \& GLEITMAN, H. (1972). A conceptual category effect in visual search: $O$ as letter or as digit. Perception \& Psychophysics, 12, 457-460.

KEREN, G. (1977, November). Additional considerations of the "category" effect. Paper presented at the Annual Meeting of the Psychonomic Society, Washington, DC.

KRUEGER, L. E. (1984). The category effect in visual search depends on physical rather than conceptual differences. Perception \& Psychophysics, 35, 558-564.

NeISSER, U. (1967). Cognitive psychology (Century Psychology Series). New York: Appleton-Century-Crofts.

SPERliNG, G., BUdianski, J., SpivaK, J. G., \& Johnson, M. C. (1971). Extremely rapid visual search: Maximum rate of scanning letters for the presence of a numeral. Science, 174, 307-311.

TREISMAN, A., \& GormiCAN, S. (1988). Feature analysis in early vision: Evidence from search asymmetries. Psychological Review, 95, 15-48.

WITTHOFT, N., \& WINAWER, J. (2006). Synesthetic colors determined by having colored refrigerator magnets in childhood. Cortex, 42, 175-183.

(Manuscript received June 22, 2005; revision accepted for publication April 1, 2006.) 